\title{
REVAMP DO SISTEMA DE CONTROLE DE UM LAMINADOR DE BARRAS E ROLOS*
}

Alexandre Schultz Rizek ${ }^{1}$ Willian Lozano de Godoy ${ }^{2}$ Ricardo Alexandre Medeiros ${ }^{3}$

\section{Resumo}

Esse projeto visou a substituição do sistema de controle instalado por um padrão RUSSULA, sistema esse que controla um laminador de barras e de rolos de 500.000 toneladas/ano na Usina Presidente Vargas da CSN em Volta Redonda, RJ/Brasil. Juntamente com o sistema de controle, RUSSULA forneceu um novo posto de operação para a cabine central, com novas telas de operação, sendo um posto "touch". Equipado com controladores Siemens S7-400, IHM Wonderware InTouch, acionamentos ABB ACS800 e DCS800, o sistema se apresentava ineficaz, exigindo a otimização do processo, aumento de produtividade, diminuição de paradas, diminuição de perdas metálicas e maior confiabilidade no controle, o último através das ferramentas RUSSULA implementadas no software, como por exemplo detecção automática de sucata, controle de tração, controle de laço, rastreamento do material no laminador, otimização de corte das tesouras e barra fantasma.

Palavras-chave: Laminador; Sistema; Controle.

\section{BAR AND WIRE ROD ROLLING MILL CONTROL SYSTEM REVAMP}

\section{Abstract}

This project has focused on changing the previous control system of a bar and wire rod rolling mill, capable of producing 500.000 tons/year, for a new RUSSULA standard, in the CSN Presidente Vargas Mill in Volta Redonda, RJ/Brazil. In addition, RUSSULA has supplied a new main control desk for the main pulpit, with new operation screens and a touch panel. Built with Siemens 57-400 controllers, Wonderware InTouch HMI, ABB ACS800 and DCS800 drives, the system showed itself inefficient, requiring process optimization, increase productivity, decrease stoppages, decrease loss metallic e control reliability, the former through RUSSULA's control improvement tools, for example automatic cobble detection, tension control, loop control, material tracking, shears cut optimization and ghost bar.

Keywords: Rolling mill; System; Control.

1 Engenheiro Eletricista, Engenheiro de Automação, Departamento Diretoria, Russula do Brasil Ltda., São Paulo, SP, Brasil.

2 Engenheiro Eletricista, Engenheiro Eletricista, Engenharia, Russula do Brasil Ltda., São Paulo, $S P$, Brasil.

3 Engenheiro Eletricista, Engenheiro Eletricista, Gerente de Vendas, Vendas, Russula do Brasil Ltda., São Paulo, SP, Brasil. 


\section{INTRODUÇÃO}

Este laminador da CSN instalado em Volta Redonda, RJ/Brasil, é um laminador com duas saídas, sendo uma delas para barras (vergalhão) e a outra para rolos. Tendo como início de operação o ano de 2012. É constituído por um forno de reaquecimento, um laminador blooming, e outro laminador dividido em 3 seções (desbaste, intermediário e pré-acabador), após o laminador existem duas saídas, uma para barras (leito de resfriamento) e outra para rolos (bloco acabador e formador de espiras).

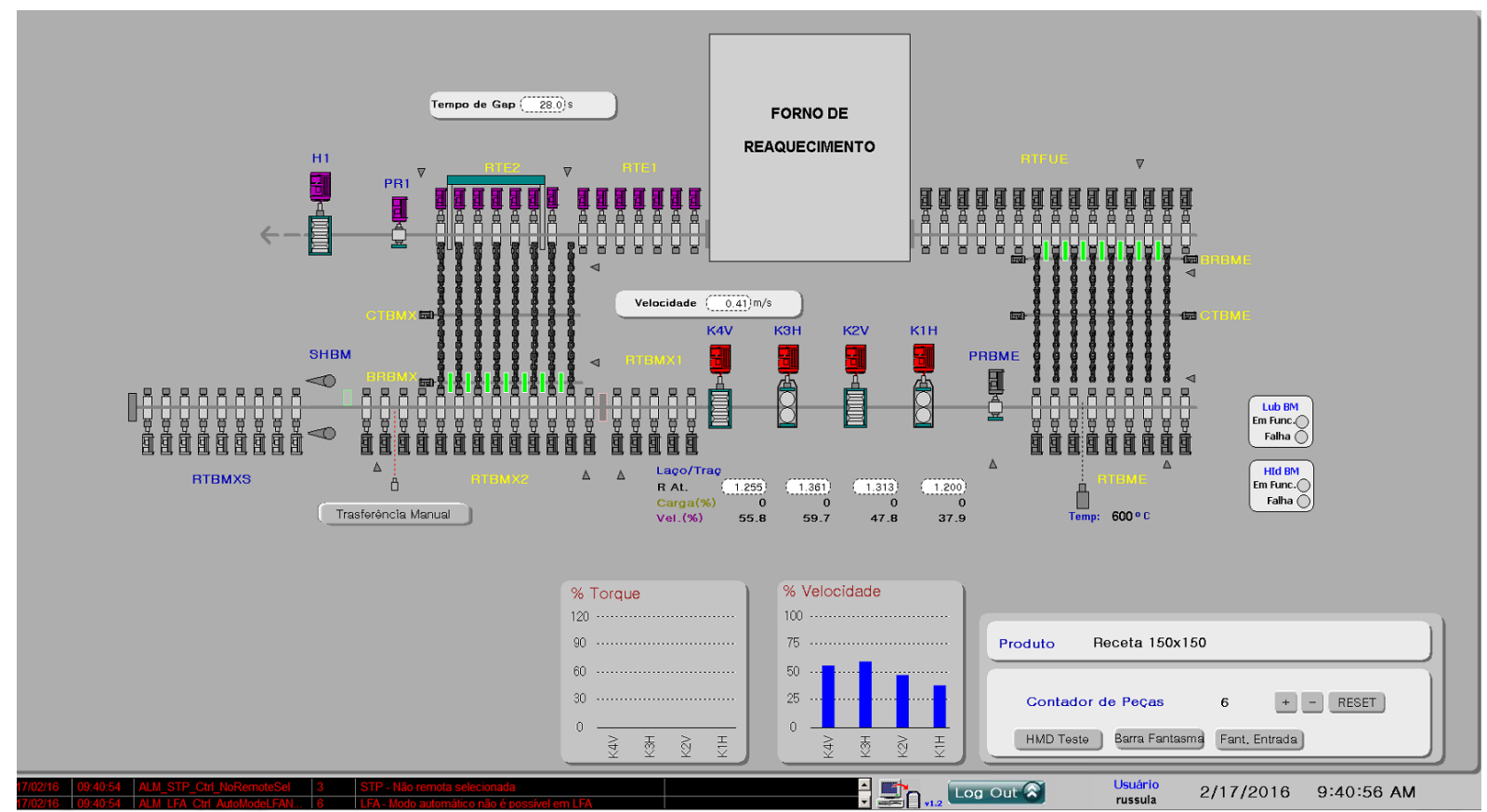

Figura 1. Laminador Blooming.

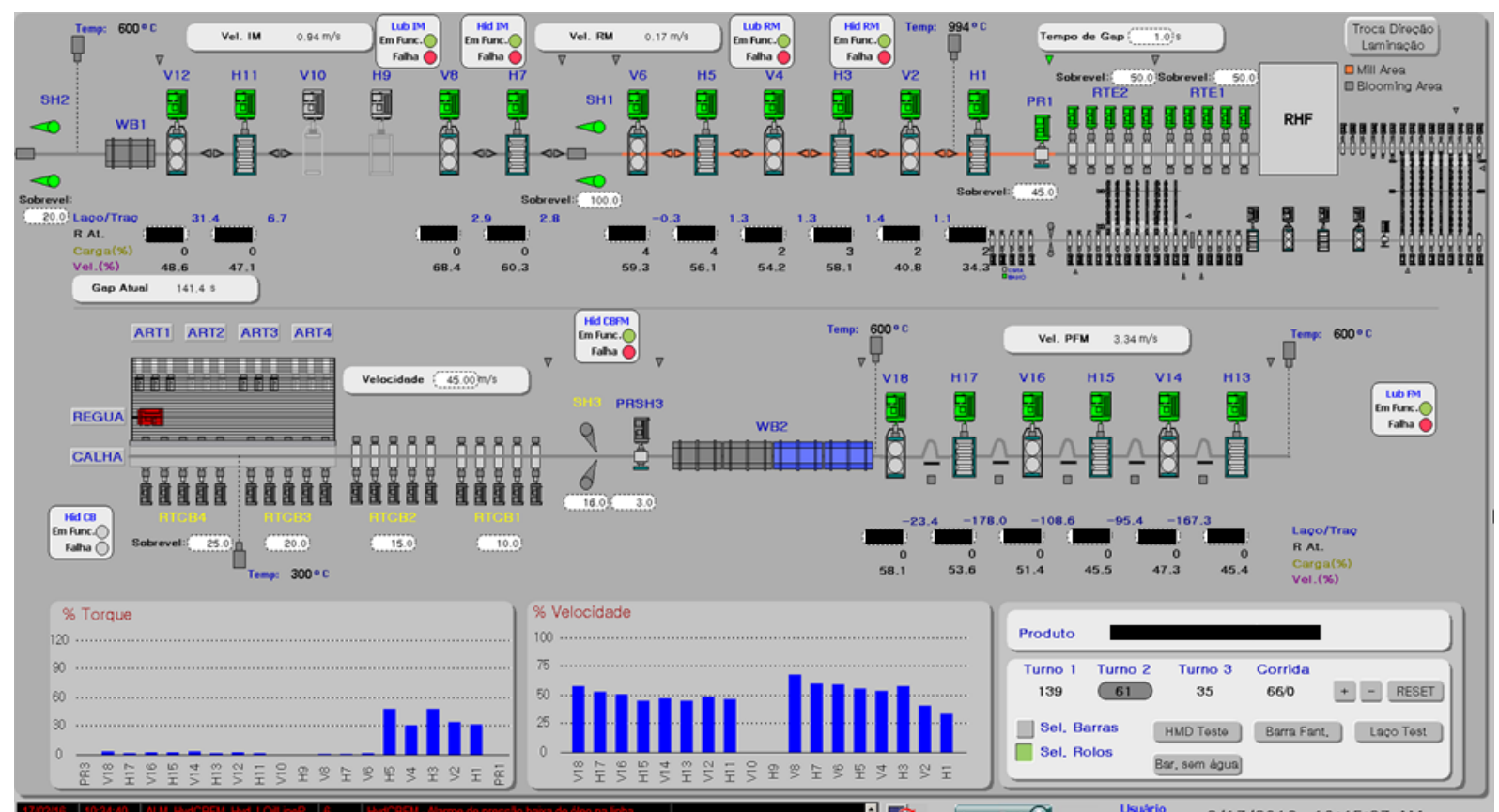

Figura 2. Laminador mais Saída de Barras. 


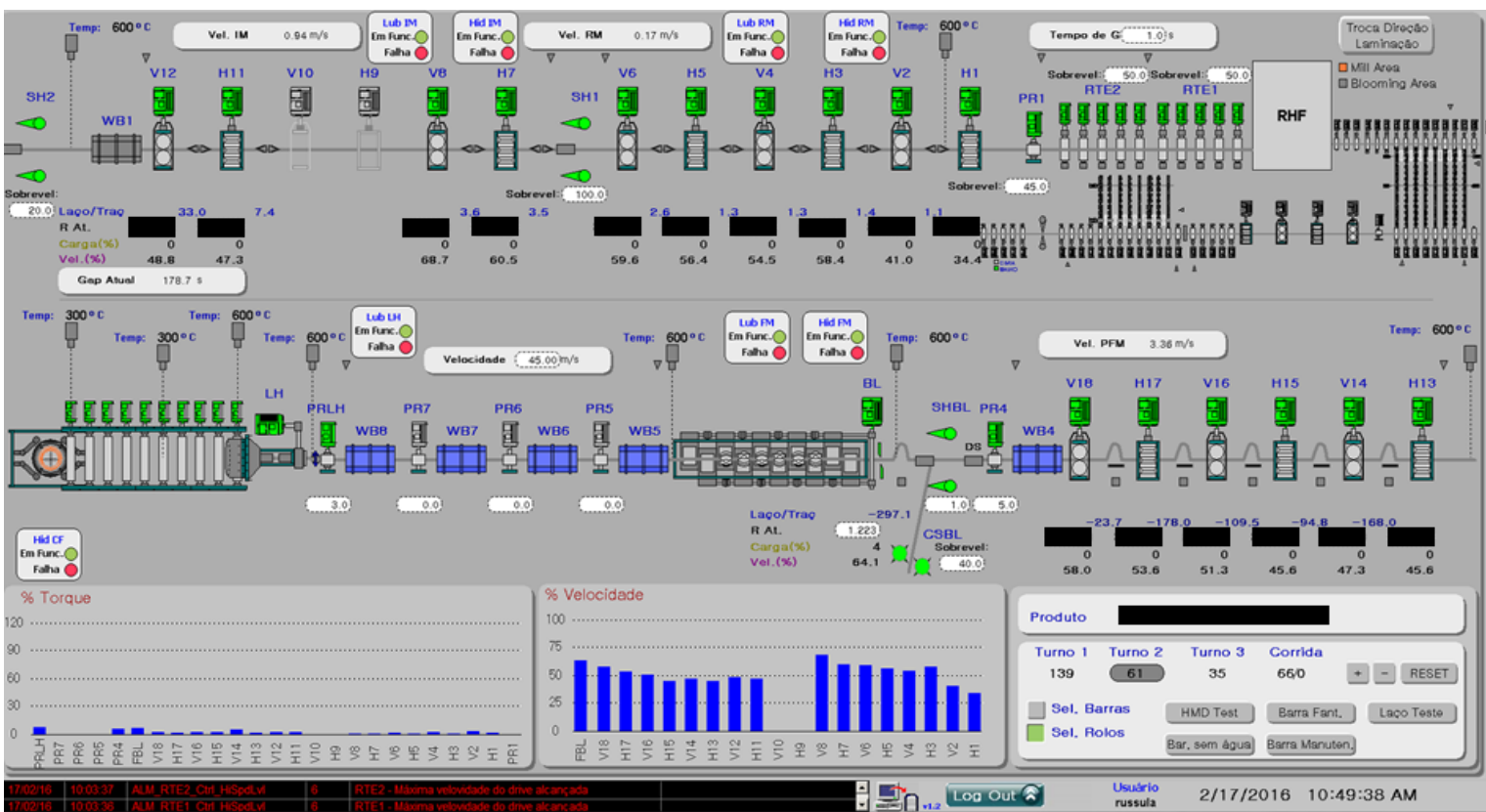

Figura 3. Laminador mais Saída de Rolos.

Buscou-se com o projeto a estabilização do processo de laminação, uma vez que o sistema antigo não era confiável.

\section{MATERIAIS E MÉTODOS}

Afim de avaliar o sistema de controle do laminador, RUSSULA realizou uma consultoria especializada na CSN. Durante duas semanas com a presença de 2 especialistas RUSSULA em processo de laminação e automação, foram levantados pontos sobre a instalação do laminador (estruturação dos equipamentos, montagem das gaiolas), seus componentes (HMDs, Loop Scanners, encoders, drives, motores), falhas do sistema de controle (excesso de tração entre gaiolas no controle MTC, não manutenção da altura do laço entre gaiolas no controle de laço, discrepância no comprimento de corte ajustado, não atingimento da máxima velocidade de produção projetada). Tendo esses itens em vista foram encontrados pontos de melhoria, como:

$\checkmark$ Controles de partida e parada de drives;

$\checkmark$ Cascata (Distribuição de Referência);

$\checkmark$ Controle de Mínima tensão;

$\checkmark$ Controle de Laço;

$\checkmark$ Detecção automática de sucata;

$\checkmark$ Posicionamento das tesouras de corte.

Após a visita foi elaborado um relatório indicando:

- Troca das HMDs;

- Troca das Loop Scanners;

- Instalação de Encoders Absolutos e Incrementais;

- Medição a laser para o blooming;

- Instalação de SIMOTION para posicionamento das tesouras;

- Cabear diretamente os sinais de HMDs e Loop Scanners ao PLC;

- Troca do púlpito principal de operação;

- Integração do sistema lba;

- Troca de equipamentos mecânicos (futuro projeto). 
Antes do comissionamento do sistema, RUSSULA realizou visitas semanais na planta afim de garantir que as instalações e pendências listadas estivessem concluídas para a posta em marcha.

O sistema de controle é composto por controladores (PLCs) Siemens S7-400, sistemas de aquisição de dados lba, acionamentos ABB ACS800 e DCS800, equipamentos estes que foram mantidos.

Além da inserção da lógica RUSSULA nos controladores, todos os acionamentos foram otimizados, afim de obter respostas mais rápidas e precisas necessárias ao processo.

No leito de resfriamento foram instalados e comissionados 4 painéis elétricos com 4 inversores ABB ACS800 para os 4 grupos de rolos alinhadores de barras.

Houve uma reestruturação na topologia da rede ethernet existente na planta, afim de obter maior velocidade na troca de informações entre PLCs, entre PLC e sistema de aquisição de dados (Iba), entre PLC e nível 2, diminuindo assim a perda de informações e geração de falhas no processo.

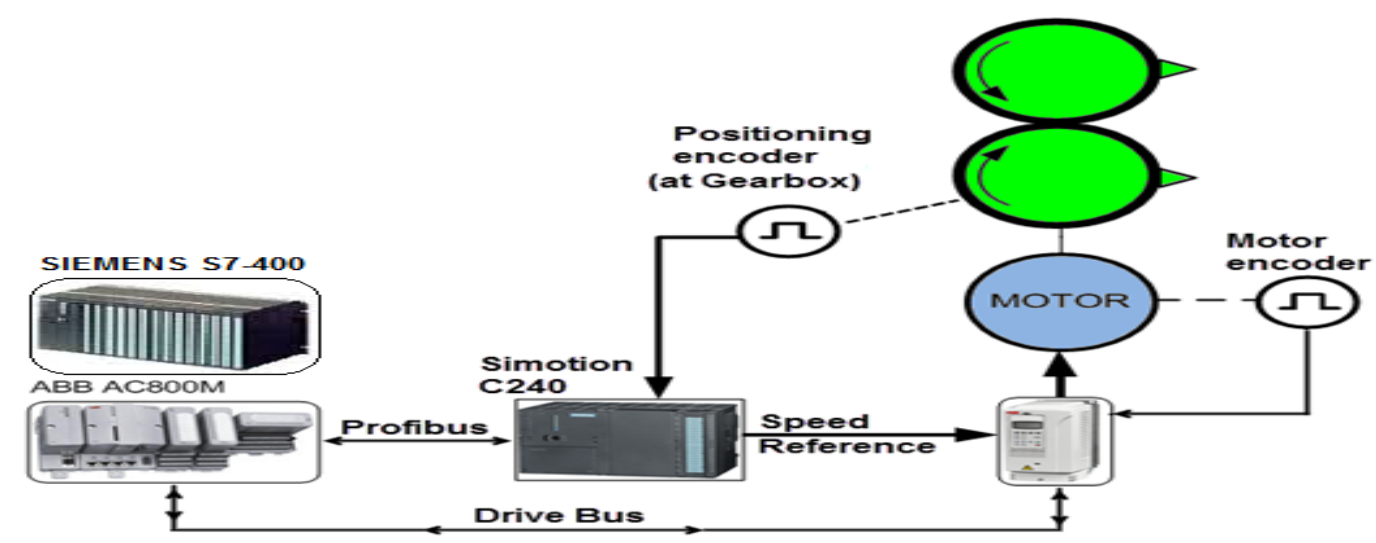

Figura 4. Controle de Posicionamento com SIMOTION.

\subsection{Principais Funções de Controle Implementadas}

\subsubsection{Receitas de Laminação}

O sistema de receitas de laminação foi projetado para permitir ao operador elaborar uma biblioteca contendo as configurações dos acionamentos de um laminador para cada uma das dimensões mais frequentes de material laminado. As receitas são identificadas e relacionadas em função da bitola do material.

O operador pode fácil e rapidamente modificar e regravar os programas de laminação para reutilização e, desta forma, aperfeiçoar gradativamente o processo de laminação. Os programas podem ser armazenados a partir de valores reais ajustados durante a laminação, ou diretamente pelo operador por meio do teclado.

Cada programa de laminação contém basicamente a configuração do laminador, "Fatores R", fatores de canal ("Groove") de cada acionamento, velocidade de produção (velocidade periférica da última cadeira de laminação), bem como sobrevelocidade e dados específicos de equipamentos auxiliares (tesouras, impulsionadores, etc.). 


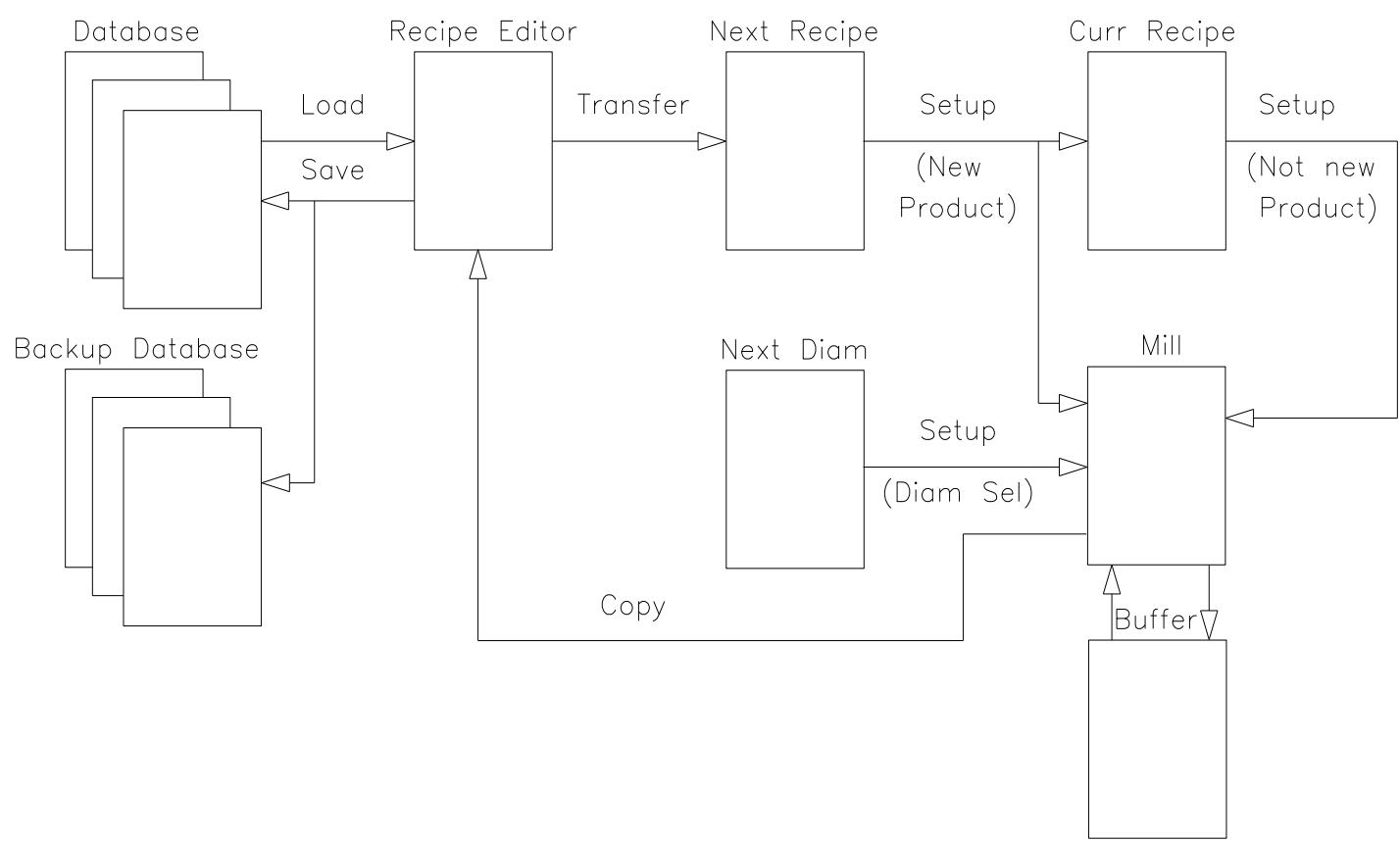

Figura 5. Estrutura de Receitas.

\subsubsection{Distribuição de Referencia}

A distribuição de referências consiste em um sistema de cascata que gera as referências de velocidade dos motores principais em todo laminador. Essa cascata age normalmente na direção da última para a primeira gaiola de laminação com o propósito de manter constante a velocidade do material nessa última gaiola.

O sistema de controle utiliza a velocidade de produção inserida pelo operador para o cálculo da rotação a ser aplicada no motor de modo a obtermos a velocidade periférica desejada na última gaiola do laminador.

Para esse cálculo necessitamos das seguintes variáveis:

- Fator de "Groove" e diâmetro do rolo, inseridos pelo operador;

- Redução do motor e valor máximo de rpm, parâmetros fixos do laminador.

A velocidade periférica de saída de uma gaiola de laminação pode ser calculada usando a seguinte fórmula:

$$
\text { VelocidadeLinear }_{[m / s]}=\frac{(\text { Dia }- \text { Groove }) * \pi * r p m}{60.000 * i}
$$

Figura 6. Cálculo Velocidade Linear.

onde:

$$
\begin{aligned}
& \text { Dia = Diâmetro do cilindro }[\mathrm{mm}] \\
& \text { Groove = Fator "Groove" }[\mathrm{mm}] \\
& \pi=3,141592 \\
& \text { rpm = velocidade do motor [rotações } / \mathrm{min}] \\
& \mathrm{i}=\text { redução caixa de engrenagem. }
\end{aligned}
$$

Para a gaiola anterior dividimos a velocidade de produção pelo Fator " $R$ " da última gaiola, obtendo assim a velocidade de saída da gaiola anterior. De posse de valor aplicamos o mesmo cálculo e variáveis acima para encontrarmos a rotação correta da penúltima gaiola. Esse procedimento é repetido até a primeira gaiola de laminação. Essa cadeia de cálculo é denominada cascata principal "upstream". 
O sistema de controle utiliza internamente a unidade métrica para os cálculos, já os valores apresentados nas telas da IHM podem ser em outro sistema (por exemplo, o sistema "imperial").

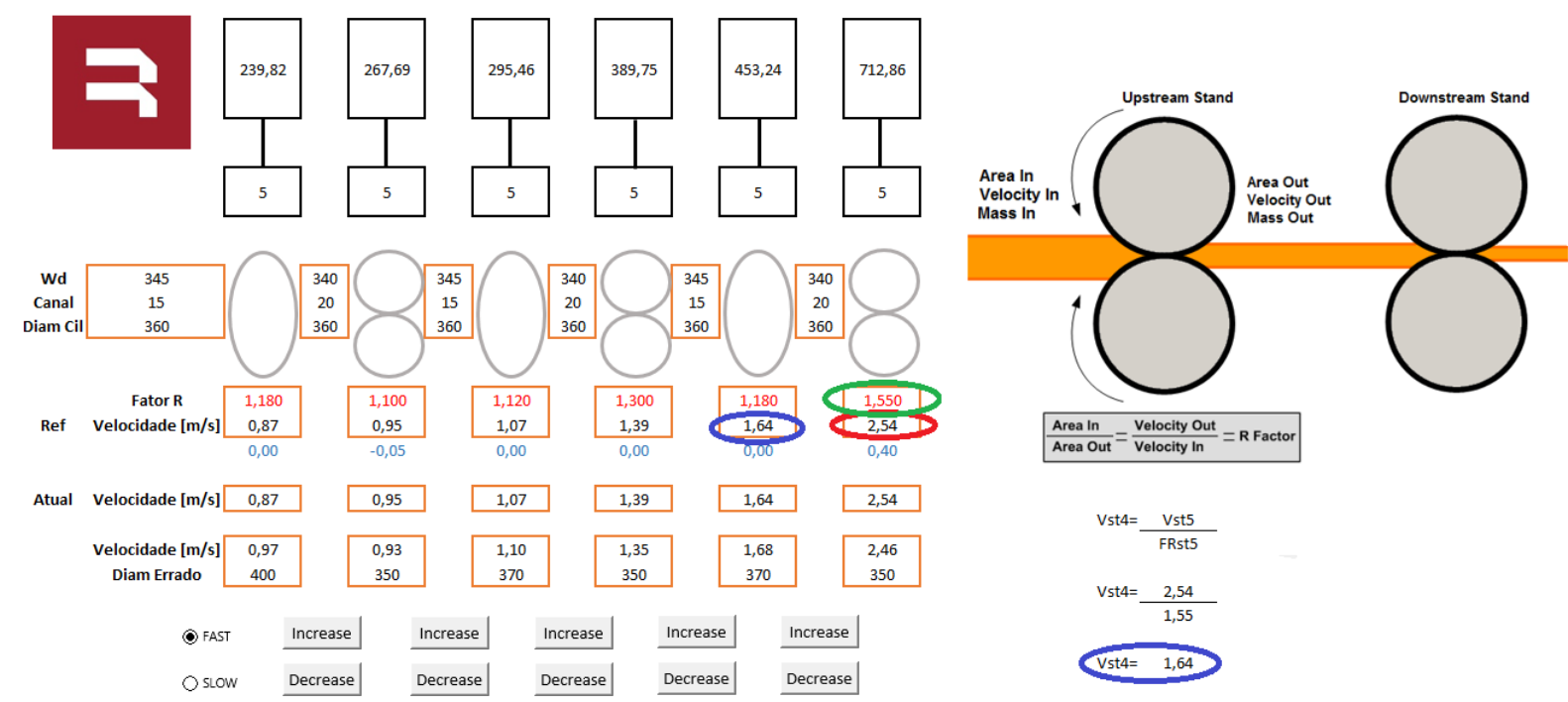

Figura 7. Fator R.

\subsubsection{Controle de Tração}

O sistema de controle de tração garante que a barra seja laminada com uma tração mínima. O operador ajusta a tração (força/área) necessária que é transformada em torque de laminação. O torque do motor da gaiola "upstream" é medido e a velocidade é ajustada de modo a manter um nível desejado do torque na gaiola.

As tomadas instantâneas do torque da gaiola "upstream" são obtidas quando a cabeça da barra está próxima à gaiola "downstream". Esse torque deve ser memorizado antes de atingir a gaiola "downstream", evitando inclusive a guia de entrada da gaiola "downstream". Este valor de torque memorizado é comparado com o torque atual da gaiola "upstream" depois da barra ter passado pela gaiola "downstream". A velocidade da gaiola "upstream" é então ajustada, pelo Fator-R, para manter o valor desejado de torque.

\subsubsection{Controle de Laço}

A finalidade principal da função de controle automático de laço é controlar e manter a laminação livre de tração, através de um laço de material entre duas gaiolas de laminação.

As informações de altura do laço e a detecção do material são fornecidas pelo sensor de laço. A medição do valor é interpretada pelo sistema de controle, fornecendo uma medição em unidade de engenharia $(\mathrm{mm})$. Este valor é compensado por uma posição de entrada do laço (ELP), parâmetro do controle do sistema, para definir a posição zero do material.

\subsubsection{Controle Manual}

$\mathrm{O}$ operador pode ajustar a velocidade de referência e o Fator-R, por meio das chaves de cascata sobre a mesa de operação. Isso irá sobrescrever qualquer controle automático e agir na direção da cascata principal. Esta correção manual pode ser utilizada para ajustar a tração em um determinador trecho onde não haja controle automático, ou para corrigir uma variação muito grande onde o controle automático possa falhar. 


\subsubsection{Rastreamento do Material}

O sistema de rastreamento acompanha a barra ao longo de sua passagem pelo laminador. O rastreamento é responsável pela ativação correta dos controles automáticos de tração e laço.

O controle de tração usa o sistema de rastreamento para coletar o ponto exato de medição do torque e habilitar o controle de tração. Já o controle de laço usa o rastreamento da cabeça e da cauda para subir e abaixar o formador de laço e iniciar o controle integral. Outras funções também usam o rastreamento para determinar o sequenciamento correto, tais como liberação do fator de compensação de impacto. A função de detecção automática de sucata também utiliza o sistema de rastreamento para indicar uma possível situação de sucata.

O sistema de rastreamento utiliza sensores de detecção de material e/ou indicação de carga no motor para acompanhar a barra no laminador. O tempo que a barra deve levar para percorrer uma determinada distância é calculado através da velocidade do material e das distâncias entre gaiolas e sensores de detecção de metal quente. A indicação de carga no motor ou o sensor de detecção são usados para verificação e aferição dos tempos calculados.

\subsubsection{Detecção Automática de Sucata}

Utilizando a posição exata da cabeça a todo momento, o sistema detecta possíveis sucatas durante a laminação automaticamente, e em função desta informação toma decisões importantes para evitar dados ao laminador ou mesmo provocar grandes paradas ou mesmo perdas de material. As decisões podem ser como, por exemplo, picotamento da barra laminada, fechamento do forno, fechamento das snap shears dos blocos, etc.

\subsubsection{Compensação de Impacto}

A compensação de impacto consiste em aumentar a velocidade da gaiola para a entrada da barra, reduzindo assim a caída de velocidade com o impacto da barra na gaiola. Após a barra está sendo laminada na gaiola, se corrige a referência de velocidade para aquela determinada pela cascata de velocidades.

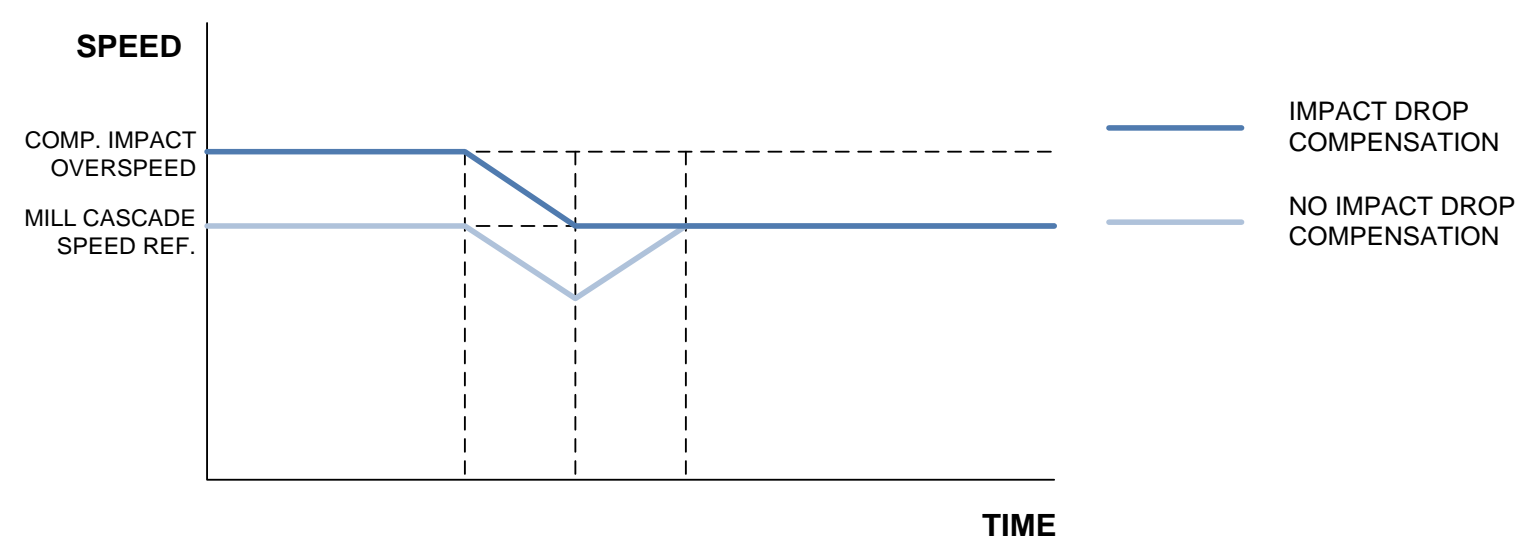

Figura 8. Compensação de Impacto.

\subsubsection{Tesouras de Desponte}

A função da tesoura de desponte é cortar um pedaço da cabeça ou da cauda, que normalmente apresentam irregularidades, em tamanhos definidos pelo operador.

O controle do disparo utiliza uma HMD (Sensor de detecção de material - hot metal detector) localizada antes da tesoura. Quando a cabeça ou a cauda da barra passa 
pelo HMD, um bloco integrador começa a calcular a distância percorrida com a velocidade do material, quando este valor atinge o tamanho calculado, o ciclo de corte é acionado (Figura 8).

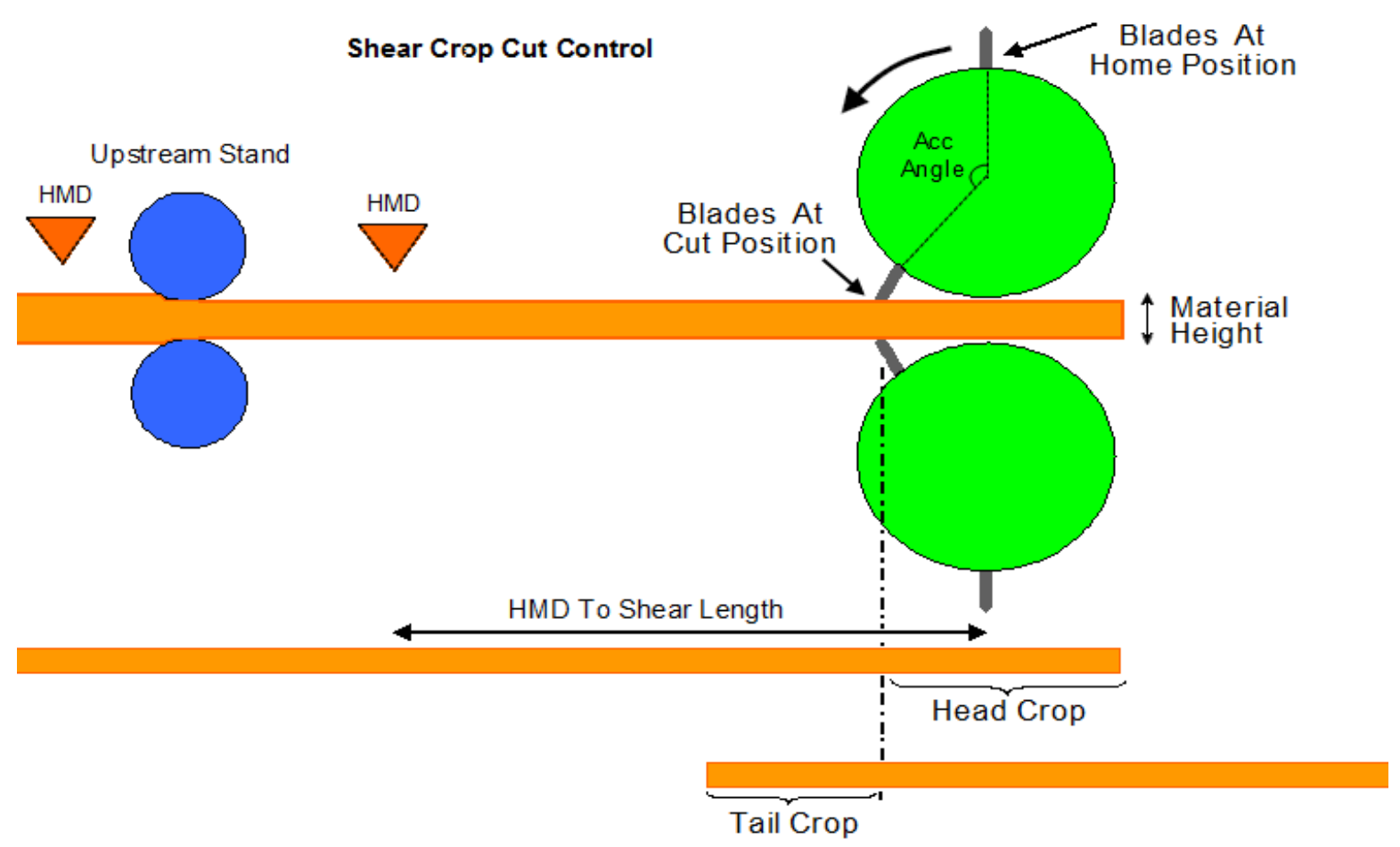

Figura 9. Despontes de Cabeça e Cauda.

$\mathrm{Na}$ IHM dispomos das seguintes opções:

1. Seleção de desponte de cabeça e/ou cauda; e

2. Comprimento do desponte de cabeça e/ou cauda.

\subsubsection{Barra Fantasma}

A barra fantasma consiste em uma ferramenta muito importante para o operador do laminador. Esta ferramenta simula efetivamente uma barra no laminador, movimentando tesouras, abrindo água, formando laços, posicionando sistemas. Com isto, o operador pode, antes de iniciar uma corrida após uma parada, certificarse que não há nenhum sistema desligado ou em mal funcionamento, evitando sucatas.

\subsection{Sistema IHM}

O software utilizado para desenvolvimento de toda interface homem máquina (IHM) foi o Wonderware InTouch, contendo dois servidores "hot stand-by". Para o sistema de receitas foi utilizado o software para banco de dados SQL. Também houve a inserção de outras ferramentas poderosas, como a supervisão geral do laminador, telas detalhadas de todas as partes do trem, migração de diversos botões físicas para as telas do IHM (reduzindo espaço e poluição visual do púlpito), adição de botões novos e a introdução de uma tela sensível ao toque, com alguns comandos que também podiam ser acessados pelo IHM convencional, caso necessário. 


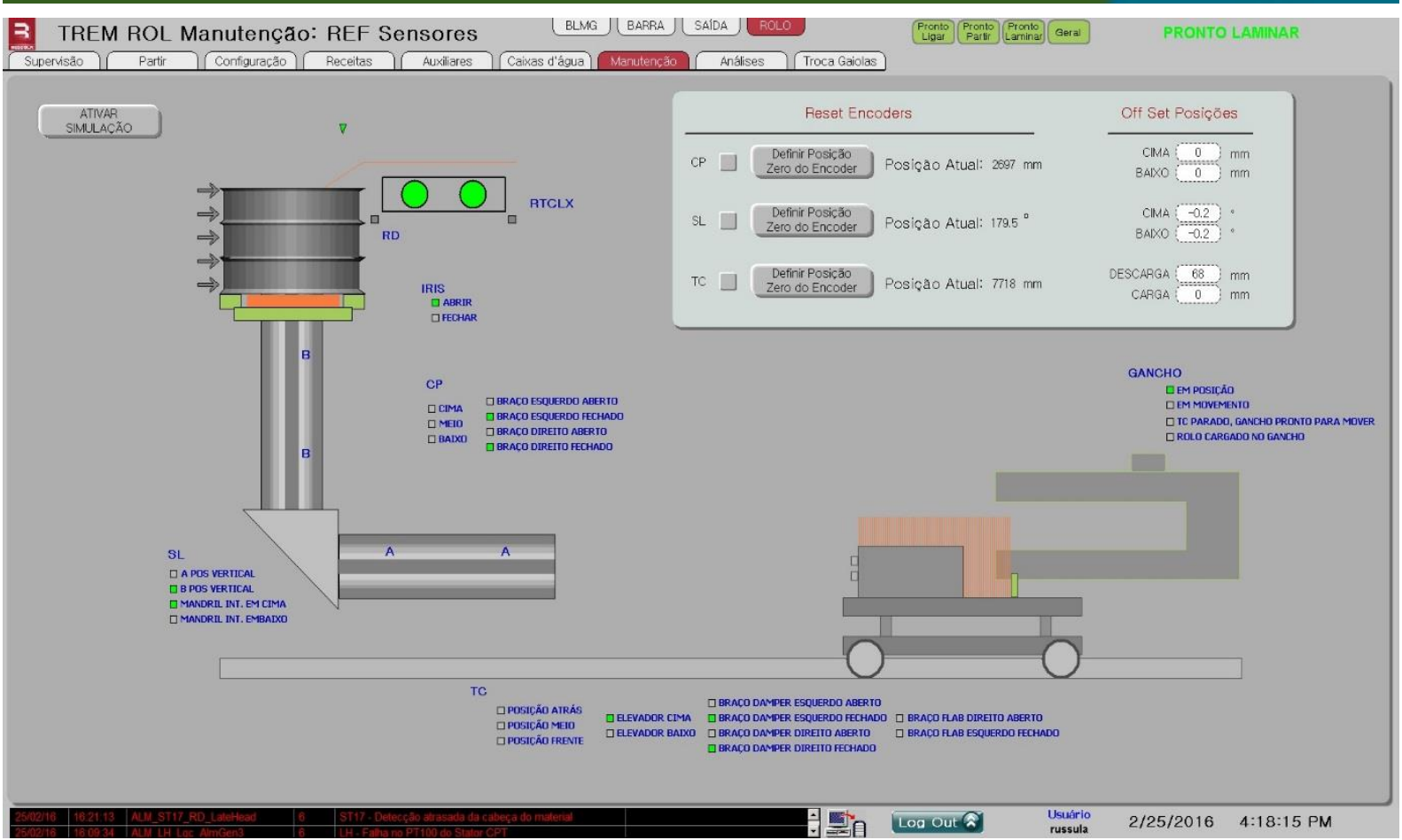

Figura 10. Tela de Supervisão de Sensores.

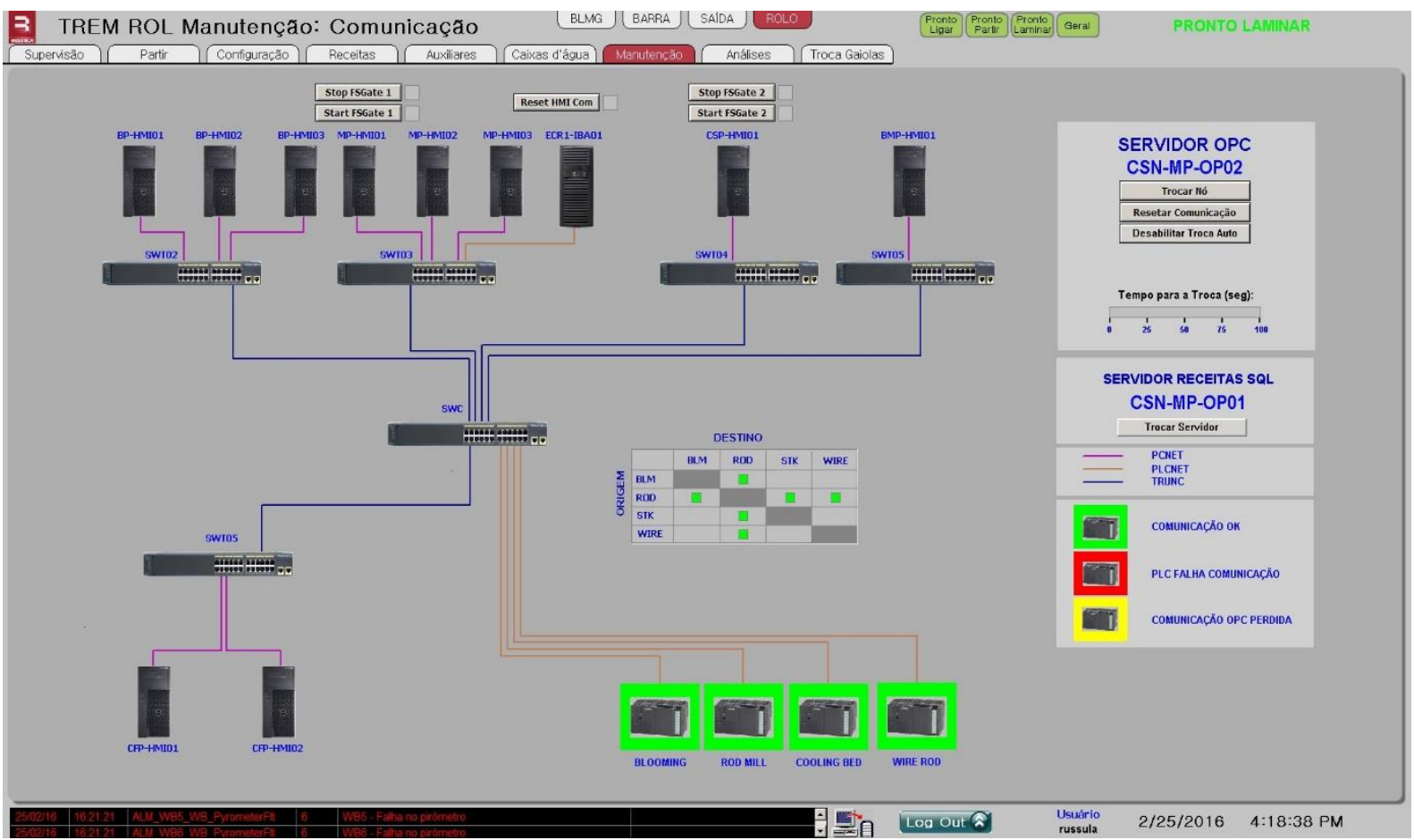

Figura 11. Tela de Supervisão da Infraestrutura de Comunicação. 


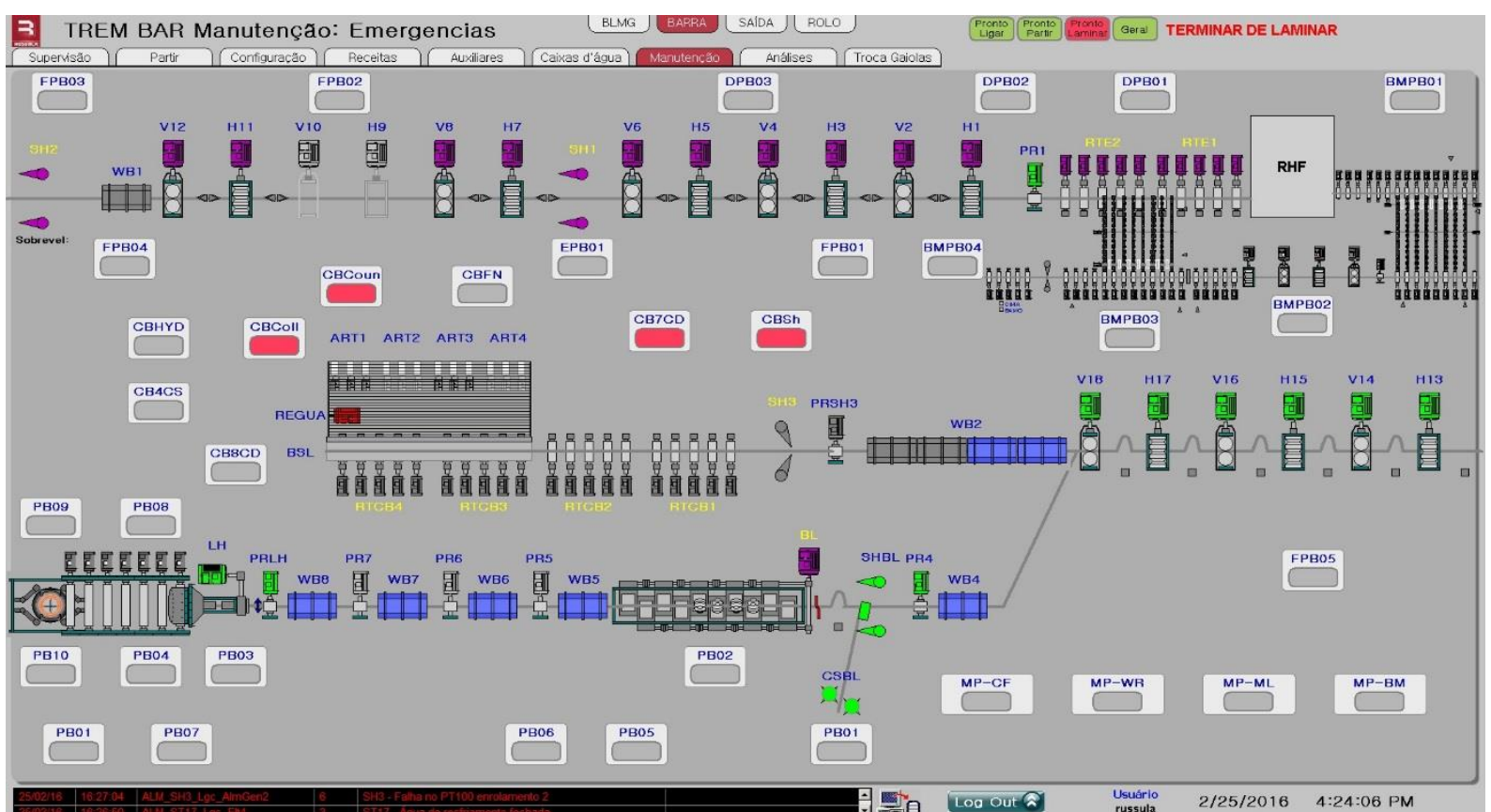

Figura 12. Tela de Supervisão das Botoeiras de Emergência.

\subsection{Sistema de Aquisição de Dados}

Um registrador de eventos IBA (PDA - Process Data Acquisition) também foi fornecido. Este registrador de eventos no laminador tem como principal função registrar informações diversas do laminador tais como: velocidades, correntes, acionamento de l/O's, etc, facilitando a solução de defeitos por parte do pessoal da manutenção. O registrador é da marca IBA, e tem uma capacidade de fazer aquisições de eventos com intervalos de até $1 \mathrm{~ms}$ por hardware e $20 \mathrm{~ms}$ for rede TCP/IP. Com esta ferramenta podemos dizer que não incorremos no mesmo problema duas vezes, pois na primeira ocorrência o sistema nos guia na identificação da causa, auxiliando na solução da falha, ou seja, é indispensável. No exemplo de tela do IBA (Figura 13), podemos ver o controle de corte de resto da tesoura.

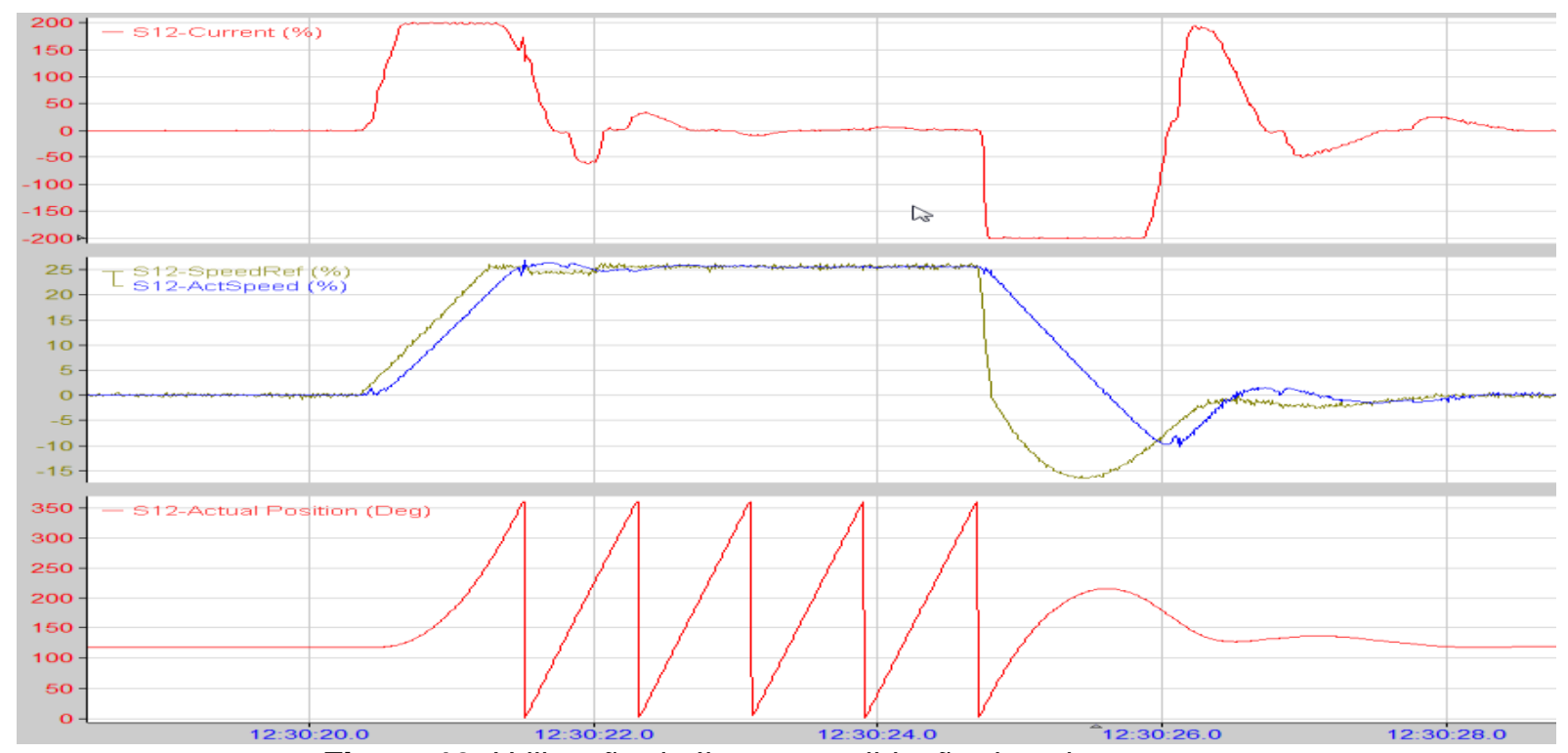

Figura 13. Utilização do Iba para validação de acionamentos. 


\subsection{Organograma}

Durante a parada, a equipe RUSSULA foi composta por um total de 13 engenheiros, esses divididos em setores pela planta, afim de otimizar recursos e diminuir o tempo de parada. Foi elaborado um planejamento em conjunto com a CSN para atender todas as metas estabelecidas, atendendo tanto solicitações comerciais quanto operacionais.

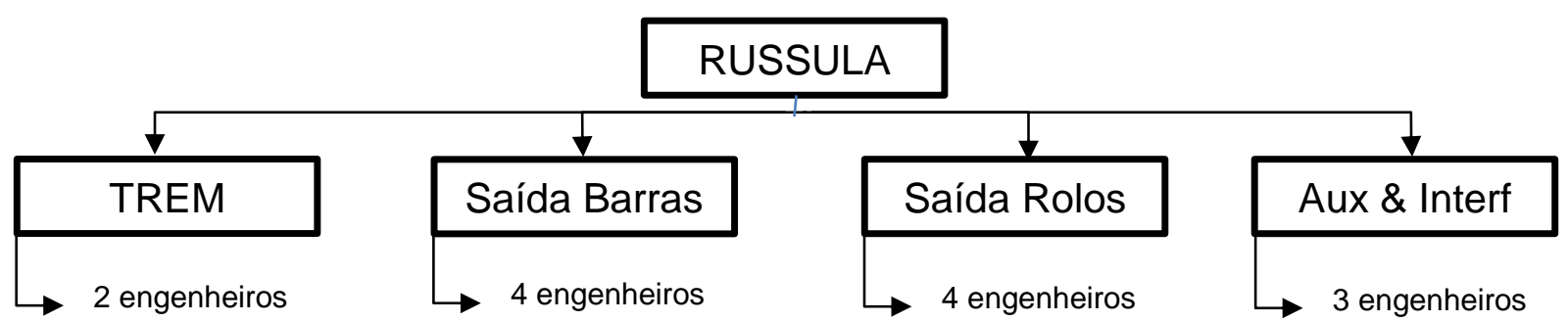

O comissionamento foi dividido em 3 etapas: Parada da saída de rolos -7 dias; Parada Trem - 10 dias; Parada da saída de barras 7 dias. Analisando o processo e o impacto de 24 dias consecutivos de parada, foi elaborada uma estratégia afim de se ter o menor tempo possível de laminador parado. Como essa planta apresenta dois tipos de saídas (barras e rolos) foi possível atuar em uma frente de trabalho enquanto a outra seguia em produção, e vice-versa. Com isso, foi possível diminuir o tempo de parada total de 24 dias para 10 dias.

Mediante planejamento e estruturação de equipes, foi possível terminar o comissionamento em 3 dias antes do prazo pré-estabelecido.

\begin{tabular}{|c|c|c|c|c|c|c|c|c|c|c|c|c|c|c|c|c|c|c|c|c|c|c|c|}
\hline \multicolumn{10}{|c|}{ Parada / Produção (dias) } \\
\hline
\end{tabular}

Figura 14. Comissionamento e Posta em Marcha.

\section{RESULTADOS E DISCUSSÃO}

Em 24 dias de comissionamento e posta em marcha, sendo somente 10 dias de completa parada, o laminador foi liberado para produção, retornando primeiramente pela saída de rolos produzindo bobinas de $10 \mathrm{~mm}$ e em seguida por uma campanha com 6mm.

Em seguida, passou-se a produzir pela saída de barras começando pela bitola $20 \mathrm{~mm}$.

Seguido pela bitola $12,5 \mathrm{~mm}$, sendo essa slit, e por fim pela bitola $25 \mathrm{~mm}$.

$\checkmark$ Melhor produção em um turno: 270 bobinas (Aproximadamente 400 toneladas);

$\checkmark$ Melhor produção em um dia: Aproximadamente 1.100 toneladas;

$\checkmark$ Aumento da velocidade de produção por rolos: de $45 \mathrm{~m} / \mathrm{s}$ no sistema antigo para $90 \mathrm{~m} / \mathrm{s}$ com sistema RUSSULA; 
$\checkmark$ Melhora no comprimento de corte de desponte de cabeça e cauda, diminuindo a perda metálica;

$\checkmark$ Automatização completa de áreas, como por exemplo corte a frio, diminuindo a necessidade de operações manuais, e consecutivamente minimizando erros;

Segundo o cliente, a laminação encontra-se em outro patamar de produção.

\section{PRÓXIMOS PASSOS}

Como novas formas de aperfeiçoamento e otimização da produção e juntamente com o relatório técnico elaborado a partir da consultoria, que identificou a dificuldade da produção de materiais com seção transversal menor, devido a não suportabilidade nas réguas móveis do leito de resfriamento, foi adquirido pela CSN da RUSSULA uma nova saída de barras contendo:

$\checkmark$ Mesa de rolos após a tesoura de corte múltiplo;

$\checkmark$ Calha de frenagem com rolos magnéticos;

$\checkmark$ Leito de resfriamento com réguas móveis.

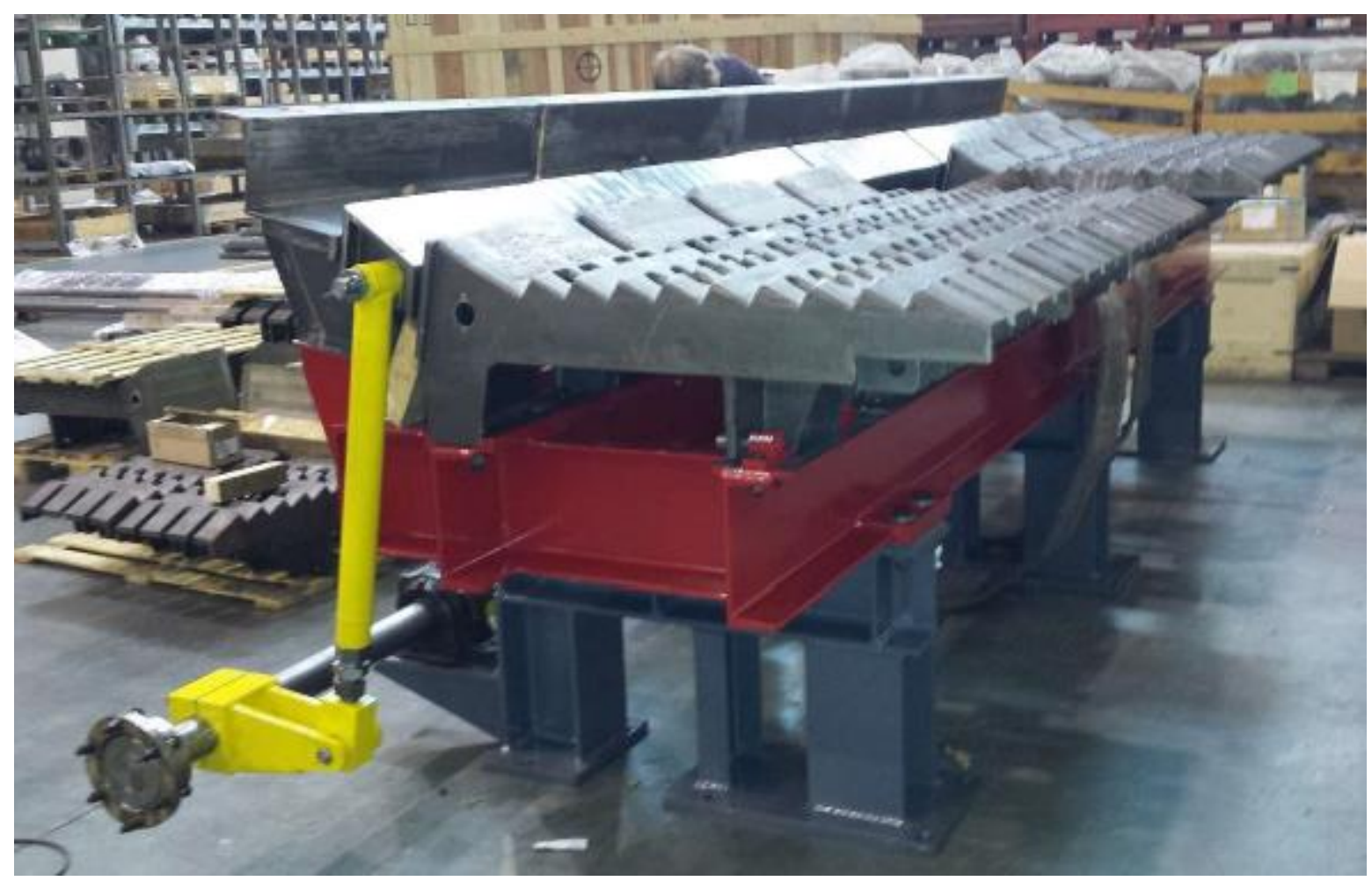

Figura 15. Leito de Resfriamento. 


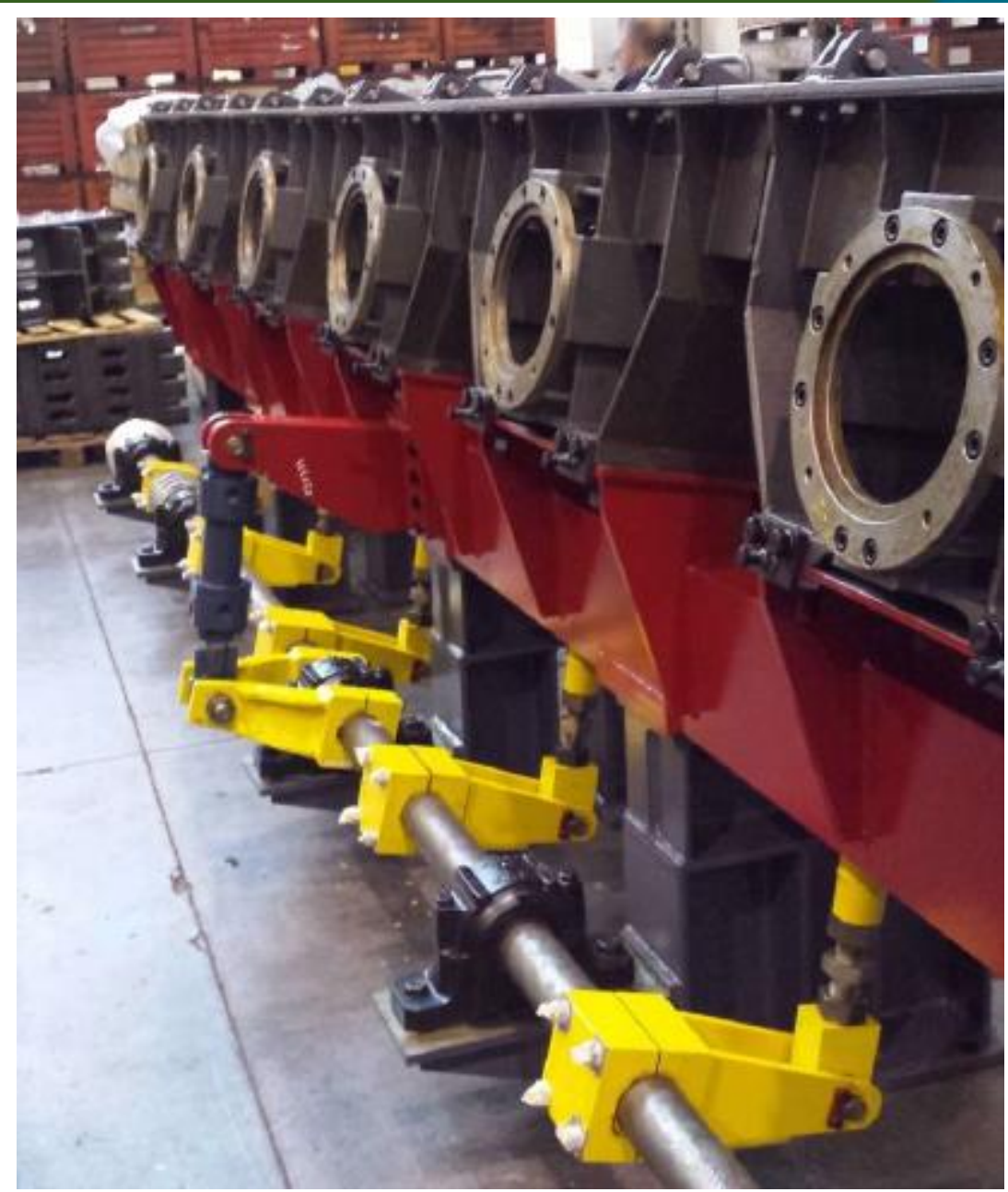

Figura 16. Leito de Resfriamento.

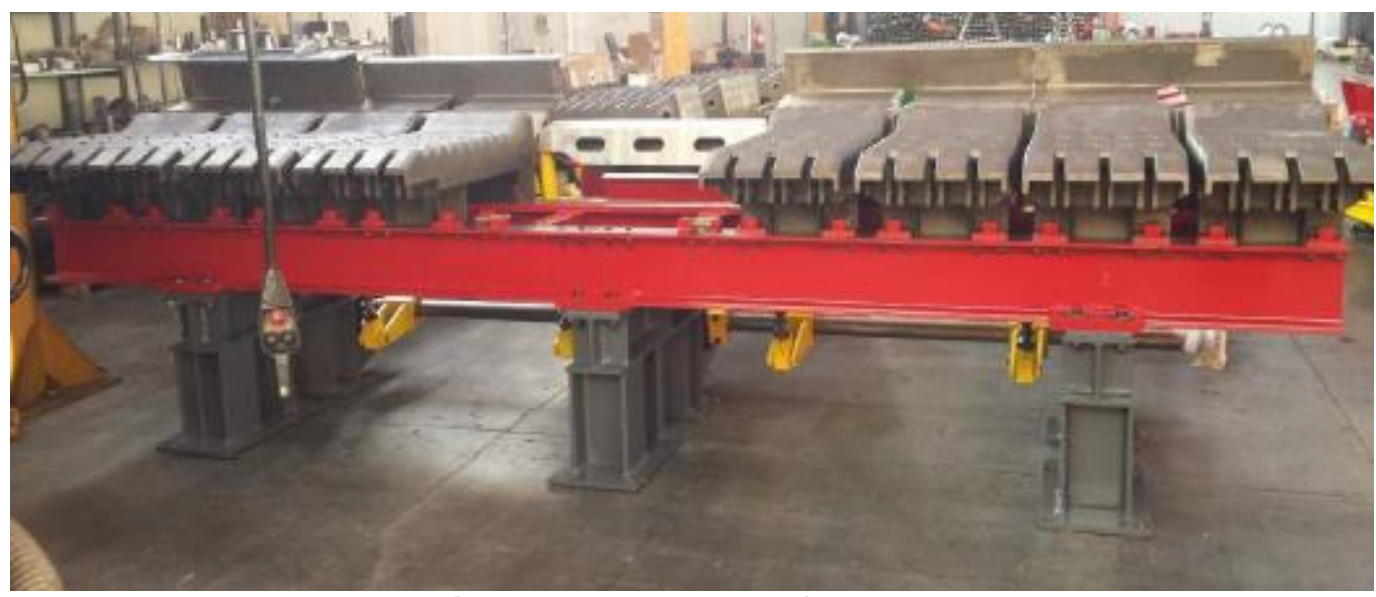

Figura 17. Leito de Resfriamento. 